\title{
Dissolution Highlights of the AAPS Annual Meeting
}

\author{
Vivian Gray ${ }^{1,3}$ and Saji Thomas ${ }^{2}$
}

e-mail:vgray@dissolutiontech.com

W ednesday, November 1, 2006, was "Dissolution Day" at the AAPS Annual Meeting. The day began with a symposium titled"The Current State of In Vivo-In Vitro Correlations and Relationships (IVIVC/R) in Pharmaceutical Technology." At lunchtime, the Hot Topic was "The Role of Dissolution in the 21st Century." In the evening, the AAPS In Vitro Release and Dissolution Testing Focus Group had its annual business meeting that included some facilitated discussion.

\section{Symposium}

There were four speakers for this symposium that was moderated by Gregg Kelly, Ph.D., from Pfizer. The first speaker was James Polli, Ph.D., from the University of Maryland. His talk, titled "Current Trends and New Developments in IVIVC/R Studies," centered on the value of obtaining a predictive IVIVC/R, which can allow for understanding the kinetics of dissolution, reduce the need for further bioequivalence studies, be compared with $\mathrm{CaCO}_{2}$ studies, and assist in selecting appropriate specifications. There are challenges to IVIVC/R when the in vivo behavior is not dissolution-ratelimited or when there are issues with the in vivo studies related to variability, drug PK, and the statistical power of the study. He also pointed out that IVIVC/R is recommended in the ICH Q8 for pharmaceutical development.

The next speaker was Colm Farrell from ICON Development Solutions, who focused on viable strategies for developing a successful IVIVC in his talk titled "Determining Whether IVIVC/R Is Difficult to Achieve Prior to Modeling." He pointed out that early planning for an IVIVC is important. Data should be generated using a number of dissolution methods as early as possible, with samples reserved for future method development. The sooner an IVIVC is established, the better, as it can be used to direct formulation development as well as support specifications and biowaiver applications.

Ed Ciolkowski, Ph.D., from Bausch and Lomb gave a presentation titled "Cross Discipline Cooperation in IVIVC/R Studies." He, too, emphasized that several dissolution methods should be developed in reserve so that if an IVIVC does not hold up with one method, it might with another. He wondered why there is not more dissolution work done in support of products. He stressed that understanding the mechanism for release is a very important aspect of Quality

\footnotetext{
${ }^{1}$ Research Editor, Dissolution Technologies, Hockessin, DE

${ }^{2}$ Associate Director, Par Pharmaceuticals, Woodcliff Lake, NJ

${ }^{3}$ Corresponding author
}

by Design and elucidating this fully is a function of the dissolution test.

The last speaker was Lawrence Yu, Ph.D., from the FDA. His talk was titled"Regulatory Experience with IVIVC/R and Biopharmaceutics Classification System (BCS)." He began his talk with a discussion of the organizational structure of the BCS Committee within CDER. This committee can advise sponsors regarding BCS and serves as a point of contact within the agency. The committee also reviews the literature for BCS classification of drugs and reviews the possibility of extensions. He stressed the importance of developing biorelavent dissolution methodology, yet acknowledged that dissolution methods presently are needed for quality control. The methodology may or may not be the same for each function. He also mentioned that he was considering looking to harmonize to only one medium for immediate-release products and maybe a series or a decision tree for media used for poorly soluble drugs.

The discussion that followed was very lengthy and informative. Most of these presentations can be found using the link http://www.aapspharmaceutica.com/meetings/ meeting.asp?id=103.

\section{Hot Topic}

This program featured two speakers, Mario Gonzales, Ph.D., from P'Kinetics International and Ajaz Hussein, Ph.D., formerly from FDA and now at Sandoz. The session was moderated by Saji Thomas, from Par Pharmaceuticals. There was a panel that consisted of Tahseen Mirza, Ph.D., from Novartis; Raman Baweja, Ph.D., from FDA; and Nicholas Capuccinno, Ph.D., from Andrx. Dr. Gonzales' presentation was from the USP viewpoint and emphasized the important role of dissolution from the bioequivalence perspective. He discussed the utility of dissolution testing in establishing an IVIVC, hence assisting in clinically relevant specifications. He also emphasized the need for USP Calibrator Tablets, now known as Performance Verification Standards, which have shown through studies to be an excellent probe, especially for vibration and vessel asymmetry issues. Dr. Hussein reiterated the need for more in-depth knowledge of the dosage form so that dissolution is not a major tool for quality control. Understanding the release mechanism and sources of product variability are paramount. Clinically relevant specifications were emphasized. The elimination of the use of calibrator tablets by having stringent mechanical calibration was stressed, and the subject of in-house standards instead of the USP standards was encouraged. This particular issue was debated during the 
discussion that followed. The problem of vibration and the adequacy of mechanical calibration for controlling and measuring this potential problem were raised.

\section{Focus Group Business Meeting}

The Business meeting was lead by Tahseen Mirza, the outgoing Chair. The newly elected Chair is Qingxi Wang, Ph.D., from Merck. There will be an election for the Chairelect. The two candidates are Alger Salt, Ph.D., from GlaxoSmithKline and Steve Mayock, from Cardinal Health. This election will be held electronically.

The activities of the past year were reviewed. A very successful workshop was held in May on the Role of Dissolution in the 21st Century (see a summary in the August 2006 issue of DT or this link http://www.dissolutiontech.com/ DTresour/200608Articles/DT200608_A04.pdf). A paper on the proceedings and discussion has been submitted for publication. A review paper on the utility of the dissolution test is in progress. Future activities are a face-to-face meeting on April 24,2007, and planning for another workshop. The agenda for the 2007 annual meeting will include three programs that are related to dissolution. It is hoped that the Modified Release and Bioequivalence Focus Groups may become more involved with our programming.

Tahseen was thanked for his excellent leadership of the focus group in its first years of existence.

\section{Focus Group Facilitated Discussion}

After the business meeting, the floor was opened up to a continued discussion of the Hot Topic issues that were presented earlier in the day. The discussions were very worthwhile and forthright. Some comments on the use of in-house standards (replacing the USP Calibrator Tablets) were that these standards could be of a "take my word for it" quality. One company had no failure problem using their own product as an in-house standard; it always passes. The point was raised that in-house standards may not be a significant probe; where is the burden of proof? What would the regulators view as justification for an appropriate inhouse standard? An in-house standard could have merit if chosen properly.

Representatives of contract labs would find working with in-house standards a burden as there may be too many if each company provided a unique tablet for qualifying instruments. Some felt the burden on companies to develop their own in-house standards is too much.

The point was raised that since USP earns money from the USP Calibrator Tablets, there would be no motivation to stop requiring them in lieu of mechanical calibration. This point was rebuffed with the fact that USP decisions along these lines come from the Expert committees, in this case, the Biopharmaceutics committee, which is composed of experts in dissolution and volunteers. These experts make the scientific decisions surrounding the use of calibrator tablets. It was also pointed out that USP was expending much time and resources to more fully characterize the USP Prednisone Tablets.

The discussion then focused on performance and operation qualifications, that is, chemical versus mechanical calibration. How often is performance qualification needed, at initial equipment setup or periodically? Some take a holistic approach that the performance qualification is the way to show confidence in the entire system. The issue was raised that mechanical calibration does not have vibration adequately controlled or measured. Also, it was pointed out that a vibration bias is usually high, generating passing results on a product that may otherwise fail the specification.

Studies on the vessels are showing that the vessels may be underspecified, and with new parameters and attributes to vessels, the price for the vessels may increase.

The question was raised as to how often and much mechanical calibration was performed daily, or after and before a run. It was also suggested that a reduced mechanical calibration (wobble, centering) before the dissolution test may be used as a system suitability test. 